\title{
sciendo
}

\section{Sagittal and Frontal Plane Gait Initiation Kinetics in Healthy, Young Subjects}

\author{
by \\ Andrew W. Smith ${ }^{1}$, Del P. Wong ${ }^{2}$
}

\begin{abstract}
The study purposes were to record the lower extremity sagittal and frontal joint moments and powers during gait initiation (GI); evaluate GI support moments in both planes; and analyze planar energy patterns in a group of 15 healthy, young adults. 3D motion and ground reaction force data were used to calculate support moments (SM) and joint moments and powers as well as center of mass (COM) kinematics. STEP1 had no visible SM. It appeared in STEP2 and, by STEP3, resembled that seen in steady-state gait. Joint moments demonstrated a similar development towards typical patterns over the three steps. Correlations of moment data between planes indicate that the frontal plane component of the SM acts to keep the COM centered. It is suggested that Winter's 1980 SM definition be extended to include both a support (sagittal) component and a centering (frontal) component. Energy was calculated for individual bursts of joint powers in both planes and each step had characteristic patterns in each plane, with patterns resembling steady-state gait appearing in the third step. Test-retest reliability (ICC range: $0.796-0.945$ ) was high with CV values in the sagittal plane $(36.6-37.5 \%)$ being less variable than in the frontal plane $(39.0-82.0 \%)$. COM kinematics revealed that acceleration peaked in STEP2 (ICC range: $0.950-0.980, C V<20.0 \%$ ). Data supported hypotheses regarding the dominance of the frontal plane power in STEP1, with substantial power coming from hip flexors. As well, powers in the sagittal plane were generally of larger magnitude than in the frontal plane.
\end{abstract}

Key words: gait initiation, joint moments, joint powers, energy.

\section{Introduction}

Gait initiation (GI) involves the shifting of body weight from double limb support during quiet standing (QS) to a single limb, moving the body forward while controlling frontal and sagittal plane forces as the body transitions to steady-state gait whereby the center of mass (COM) velocity is relatively constant. With the feet side-by-side prior to GI, the initial intended swing limb is unable to develop 'push-off' energy normally seen in steady-state gait. Thus, energy for the first step must come from other sources, including passive gravitational forward lean and active hip flexor contraction to pull the swing leg forward. As opposed to the predominately sagittal motion of steady-state gait, GI begins with the lateral shifting of the COM, which cannot be too lateral or too fast as this may result in a loss of balance, possibly leading to a fall (Rogers et al., 2001; Rogers and Mille, 2003).

From a neuromuscular perspective, when a subject commits to begin walking, soleus activity is inhibited followed immediately by activation of the tibialis anterior of both legs, which allows the center of pressure (COP) to move backward (Crenna and Frigo, 1991; Brunt et al., 1999, 2000). Concurrently, hip abductors are inhibited on the initial stance limb and activated on the ipsilateral limb, such that the COP shifts briefly towards the stance limb (Carlsöö, 1966). Paradoxically, this pattern results in the initial swing limb being loaded momentarily while the contralateral limb is unloaded, the intention of

\footnotetext{
1 - Motion Analysis Research Center, Samuel Merritt University, Oakland, CA.

2 - titi Sport Technology, Shenzhen, China.
} 
which is to allow the COP to accelerate the COM toward the contralateral limb. This results in unloading the initial swing limb so that the first step of GI may take place (Jian et al., 1993). Subsequently, the COP is driven medially during stance limb loading, and forward along the stance foot as the swing limb is lifted upward and forward (Corbeil and Anaka, 2011; Halliday et al. 1998). During the first step of GI, hip abductors of the stance limb act to prevent the pelvis from dropping on the side of the intended swing limb (Carlsöö, 1966).

Previous research has considered different numbers of steps for studying GI, including one step (Breniere and Do, 1986), two steps (Jian et al., 1993), three steps (Mann et al., 1979; Miff et al., 2008; Miller and Verstraete, 1996a, 1999), and four steps (Muir et al., 2014) depending upon what parameter of gait was being considered to define steady-state gait. For this study, we wanted to examine the entire process of moving from a stationary position to a point where most gait parameters reflected steady-state gait. Thus, we considered steadystate gait to occur at the end of the third step, in our case, after two left steps and one right step (LR-L). Our variables of interest in this study were sagittal and frontal plane GI kinetics; specifically, joint moments of force and powers, and the related support moment (Winter, 1980) along with joint mechanical energy patterns.

Winter's Support Moment Principle

In 1980, Winter proposed the principle of the overall support moment, i.e., the algebraic summation of sagittal plane lower extremity joint extensor moments of force during gait (Winter, 1980). The principle was based on the concept that the central nervous system (CNS) does not simply control individual joint moments of force; rather, the entire lower extremity acts as a single unit with respect to support against gravity, particularly during the single support phase of gait where the collapse of the limb would be disastrous. The support moment allows the CNS considerable flexibility in adapting joint motion patterns of the lower extremity hip and knee moments, depending upon such circumstances as pain avoidance, changes in terrain, speed, aging, and so forth.

Since 1980, many researchers have included support moment data in numerous published studies. These include studies of walking and running in both healthy and injured/disabled populations (Chmielewski et al., 2001; Hebert et al., 1994; McNee et al., 2004; Sanderson and Martin, 1996) as well as stair climbing and slope walking (Hong et al., 2014a, 2014b; Lay et al., 2006; Mandeville et al., 2007; Novak and Brouwer, 2011, 2013). In fact, the widespread acceptance of the concept of the support moment is clear from the fact that its use has gone beyond the original paradigm of gait to such topics as squatting (Hwang and Kim, 2009; Liu et al., 2010; Roos et al., 2014), muscle fatigue (Orishimo and Kremenic, 2006), osteoarthritis (Liu et al., 2014; McQuade and de Oliveira, 2011), cerebellar ataxias (Serrao et al., 2012), and methodological studies (McCaw et al., 2013). The principle has even been extended to non-human gait studies (Brown et al., 2003; Burton et al., 2011). Thus, the concept of summing lower extremity moments is so well established as support moment that similar mathematical operations on lower limb joint moments during non-gait activities make reference to Winter's principle (Perell et al., 2002).

Joint Power and Mechanical Energy in Gait Initiation

From the perspective mechanical energy, GI has been described as the the changing of the body's energy state from primarily potential energy during QS to the states of both kinetic and potential energy during the first three steps (Miller and Verstraete, 1999). Previous energy analyses of GI have considered segmental components and total body energy throughout each step calculated from anthropometric and kinematic data (Miller and Verstraete, 1996a, 1999). In the present study, we employed a kinetic analysis of joint power with time integration to determine joint energy patterns. This allowed us to fully describe control strategies employed by subjects, revealing when energy was being absorbed or generated. In turn, this identified when muscles were acting eccentrically and concentrically. Sagittal and frontal plane analyses of GI joint powers offer the advantage over kinematics-based techniques (Miller and Verstraete, 1996b) in identifying phases of energy generation, absorption, and transfers across joints, allowing us to understand more fully the GI process.

GI is a transitional state between QS and 
steady-state gait through which healthy individuals pass normally with ease. However, for individuals with neurological conditions such as multiple sclerosis or Parkinson's disease, movement transition states such as GI present unique neuromuscular control challenges. In the former population, the requirement of accelerating the body, both laterally and forward during GI, increases the risk of falls (Callisaya et al., 2016; Wajda et al., 2015), while in the latter population, the study of the energetics of GI may be of particular interest as it relates to the phenomenon of 'freezing' of gait (Delval et al., 2014). In addition to our current understanding of GI kinematics, center of pressure (COP) and electromyographic (EMG) patterns, kinetics-based analyses, as opposed to the study of kinematics alone, offer a more complete picture of how healthy individuals typically transition through GI, and may provide additional clues for developing novel strategies in disabled populations to prevent falls and overcome motor blocks that occur in GI.

To date, there have been virtually no three-dimensional (3D) kinetic analyses of GI, and none reporting energy utilization from a power analysis point of view or that has examined the frontal plane component of the overall support moment during GI. Our methodology allows one to explore the possibility that the support moment may play an additional role in the frontal plane. Thus, the purposes of this study were to: a) record the lower extremity joint moments and powers in the sagittal and frontal planes during the first three steps of GI; b) evaluate support moments in both planes; and c) analyze planar energy patterns during GI in a group of healthy, young adults. Hypotheses

1. The sagittal plane support moment would be like that previously reported and would be evident following the first step.

2. There would be a differentiation in the roles of the sagittal and frontal planes support moment, respectively.

3. Sagittal powers would have little contribution during the initial transfer of weight in the stance phase of the first step.

4. The energies in the sagittal plane would be significantly larger than those of the frontal plane particularly for the hip in the first step forward.

5. By the third step of GI, all kinetic variables would be like those seen in steady-state gait.

\section{Methods}

Participants

Nine females and six males with no reported neurological or musculoskeletal conditions were convenience-sampled for the study. The study was conducted according to the Declaration of Helsinki and the protocol was fully approved by the University of Auckland Human Research Ethics Committee before the commencement of the assessments. Prior to data collection, subjects' mass and height were recorded and body mass index (BMI) was calculated (Mean \pm SD mass: $73.96 \pm 16.67 \mathrm{~kg}$; height: $1.75 \pm 0.10 \mathrm{~m}$; BMI: $24.09 \pm 4.23 \mathrm{~kg} / \mathrm{m}^{2}$ ). Female subjects had significantly smaller values for mass $(t=5.004, p<0.001)$, height $(t=3.256, p=$ $0.006)$, and BMI $(\mathrm{t}=2.364, p=0.034)$.

Data Collection

Subjects stood with their feet shoulderwidth apart on two adjacent force platforms (FP1 \& FP3) for a minimum of $5 \mathrm{~s}$ to stabilize their motion, and self-initiated walking with the left foot landing on a third force platform (FP2) (Figure 1A). They continued walking for a minimum of $4 \mathrm{~m}$. A total of five GI trials were recorded for each subject. The only constraint on the subjects was that all trials began with the left leg; otherwise, they were free to initiate walking as they normally would do outside the laboratory.

Ground reaction force (GRF) data were obtained from three force platforms (Model 406008 (x 2) and Model 6090, Bertec Corporation, Columbus $\mathrm{OH}$, USA). Kinematic data were obtained using a synchronized eight-camera motion capture system (VICON MX). A 21marker model, including 15 markers comprising the 3D lower limb model (VICON Plug-In Gait), along with 3 additional upper body markers (shoulder, elbow and wrist) used with the 3D data to calculate the COM, defined an 11-segment model (6 lower limb segments, head and trunk, and 4 upper extremity segments). Kinematic data were filtered using quintic spline functions (Veldpaus et al., 1988). Kinematic and GRF data were time synchronized via A/D sampling by the data collection computer at 100 and $1000 \mathrm{~Hz}$, 
respectively.

Data Analysis

Joint kinetic data of the lower extremity were calculated using the 3D marker and GRF data. Moments and powers were normalized to subject's individual body mass, i.e, $\mathrm{Nm} / \mathrm{kg}$ and $\mathrm{W} / \mathrm{kg}$, respectively. The data were analyzed for three successive steps: STEP1 (left), STEP2 (right), and STEP3 (left). Furthermore, the data were time-normalized, i.e., 0 to $100 \%$ of GI, with 6 gait events identified (Figure 1B), and ensemble averaged across trials and between subjects. For standardization, the start of the 3-step cycle was defined to have occurred $0.5 \mathrm{~s}$ prior to STEP1 toeoff and continued through STEP2 until the 2nd left heel contact at the end of STEP3. As no timenormalized gait events data for three successive GI steps have been published previously, the normalized GI cycle events used in the present study were determined by calculating the percentage of the total GI time where heel contacts and toe offs occurred for each of the 15 subjects, then averaging these data to determine the percent values shown in Figure 1B. The normalization was done for plotting purposes and for calculating means and coefficients of variation $(\mathrm{CV})$.

\section{Data Analyses}

Following the method of Winter (1980), the support moment in the sagittal plane was calculated by summing the hip, knee, and ankle extensor moments. This methodology was adapted for frontal plane moments of force by summing the abductor/evertor moments of the hip, knee, and ankle, respectively. Hip, knee, and ankle joint power bursts in both planes were identified and labeled according to the convention published by Winter (1983a): hip H1 to H3, knee $\mathrm{K} 1$ to $\mathrm{K} 4$, and ankle A1 and A2. Additional bursts were consistently present in the frontal plane, resulting in a modification of the labeling system of the ankle, e.g., A1A, A1B. All power bursts were given unique labels for statistical analysis purposes, e.g., $\mathrm{S} 3 \mathrm{H} 3$ is the 3rd hip power burst in STEP3. Energy generation and absorption were quantified by integrating the joint powers using a trapezoidal technique (McFadyen and Carnahan, 1997) in the frontal and sagittal planes. Velocity and acceleration COM data were computed from the resultant COM position data using the central differences method (Winter, 1990).
Statistical Analysis

A $3 \times 2 \times 3$ ANOVA examined the main and interaction effects of step, plane, and joint, respectively, on energy absorption and energy generation. Post hoc tests with Bonferroni correction were conducted where appropriate. Paired-sample t-tests were used in cases where there were two groups of comparison. Pearson product moment correlation coefficients were calculated to determine the relationship of planar joint moments and support moment, and planar joint powers within STEP1, STEP2, and STEP3. The magnitude of the correlations was determined using the modified scale by Hopkins (Hopkins, 2000): $\mathrm{r}<0.1$, trivial; $0.1-0.3$, low; $>0.3$ - 0.5, moderate; $>0.5-0.7$, high; $>0.7-0.9$, very high; > 0.9, nearly perfect; and 1.0, perfect. The level of significance was set a priori at $p \leq 0.05$. The test-retest reliability of the energy values was assessed by intraclass correlation coefficients (ICCs), whereas variance was assessed by coefficients of variation (CVs).

\section{Results}

Joint and Support Moments

Ensemble averaged joint moments and support moment data (mean: solid line; \pm 1 SD: dotted lines) are shown in Figure 2, with sagittal plane data on the left and frontal plane data on the right along with coefficient of variation $(\mathrm{CV})$ data. To facilitate understanding the natural sequence of GI, STEP1 and STEP3 are plotted continuously (left leg) with STEP2 (right leg) plotted underneath for each joint in each plane. Vertical dotted lines show the sub-phases of the 3step GI cycle as defined in Figure 1B.

Sagittal plane:

The typical support moment pattern, i.e., predominately extensors during stance and zero during swing, seen in steady-state gait was not evident in STEP1. During STEP2, there was a gradual increase in magnitude during the stance phase of the support moment as the COM began to move away from the starting point. By STEP3, there was positive (extensor) activity during stance and slightly negative or zero support moment during swing, which was more or less typical (Winter, 1980). At the lower extremity joints, the magnitudes of the moments in STEP1 were relatively small, with most of the activity occurring in STEP2 and STEP3 at the ankle. 
Typical support moment patterns at the hip and knee were not evident until STEP3.

Frontal plane:

As that seen in the sagittal plane, there was no discernable support moment in STEP1, but both STEP2 and STEP3 fully developed abductor support moment patterns, with average magnitudes larger than that seen in the sagittal plane. In fact, the support moment in STEP2 began immediately, which was expected since there was only a brief $(0.5 \mathrm{~s})$ stance phase in STEP1. However, where the largest joint moments occurred at the ankle in the sagittal plane, the hip moments in STEP2 and STEP3 were larger than the moments at the knee or ankle.

\section{Planar Comparisons of Joint and Support Moments}

Relationships between sagittal and frontal planes' joint and support moment patterns were calculated using the Pearson product-moment (Table 1). Virtually all correlations were statistically significant, with the exceptions of the hip moment in STEP2 and the knee moment in STEP3. The significant correlations ranged from 0.23 (low) to 0.93 (nearly perfect). Support moment correlations were very high for STEP1 ( $\mathrm{r}$ $=-0.82)$ and STEP2 $(r=0.80)$, and nearly perfect for STEP3 $(r=0.93)$, while the corresponding correlations for the hip were moderate for STEP1 $(\mathrm{r}=-0.45)$, low for STEP2 $(\mathrm{r}=-0.16$, not significant), and moderate for STEP3 $(r=-0.34)$. Correlations for the knee were moderate for STEP1 $(r=0.32)$ and low for STEP $2(r=-0.23)$ and STEP3 $(r=0.10$, not significant), while for the ankle it was nearly perfect for STEP1 $(\mathrm{r}=-0.92)$, very high for STEP2 $(r=0.87)$, and high for STEP3 $(\mathrm{r}=0.68)$.

Joint Powers

Ensemble averaged joint power data (mean: solid line; \pm SD: dotted lines) are shown in Figure 2, with sagittal plane data on the left and frontal plane data on the right. Mean energy burst data are labelled along with coefficient of variation $(\mathrm{CV})$ data. STEP1 and STEP3 are plotted together (left leg) with STEP2 (right leg) plotted underneath for each joint in each plane. Vertical dotted lines correspond to the main gait events (Figure 1B).

Significant main effects were found in the step $(\mathrm{F}=49.2, p<0.001$, partial eta squared $=0.35)$, plane $(\mathrm{F}=177.6, p<0.001$, partial eta squared $=$ $0.66)$, and joint $(\mathrm{F}=41.8, p<0.001$, partial eta squared $=0.32)$. Significant interaction effects were found between step and joint $(\mathrm{F}=4.0, p<$ 0.01 , partial eta squared $=0.04)$, step and plane $(\mathrm{F}$ $=49.2, p<0.001$, partial eta squared $=0.35)$, joint and plane $(\mathrm{F}=53.5, p<0.001$, partial eta squared $=$ $0.37)$, and step, joint and plane ( $\mathrm{F}=6.4, p<0.01$, partial eta squared $=0.07$ ).

Differences between Steps

ANOVA results revealed that energy absorption patterns during the three steps were significantly different. As shown in Table 1, in the sagittal plane, the energy absorption at the ankle joint during STEP1 was significantly reduced compared to STEP2 and STEP3 $(p<0.05)$, and that of STEP2 was also significantly less than STEP3 ( $p$ $<0.05)$. Likewise, energy absorption at the knee joint in the sagittal plane was significantly less in STEP2 as compared to STEP3 $(p<0.05)$. In the frontal plane, only the knee joint energy absorption during STEP2 was significantly different from STEP3 $(p<0.05)$.

Similarly, ANOVA results revealed that energy generation patterns during the three steps were significantly different. The energy generation in the sagittal plane at the ankle joint during STEP2 was significantly larger compared to STEP1 and STEP3 $(p<0.05)$ (Table 1). STEP2 had significantly more knee energy generation than STEP3 $(p<0.05)$; in turn STEP3 had significantly higher hip energy generation than STEP1 $(p<0.05)$. In the frontal plane, STEP2 had significantly more ankle and knee energy generation than STEP1 $(p<0.05)$, as well as larger hip energy generation than STEP3 $(p<0.05)$.

Energy Differences between Joints

ANOVA results demonstrate that energy absorption patterns are also significantly different between joints. Specifically, the energy absorption at hip joint in the sagittal plane is significantly more than ankle and knee joints during STEP3 (Table 2). In the frontal plane, ankle energy absorption was significantly smaller than knee and hip joints $(p<0.05)$ during STEP2. Ankle energy absorption was significantly smaller than hip joint during STEP3.

At the same time, energy generation between the joints was significantly different. Specifically, during STEP1, hip energy generation was significantly larger than ankle in the sagittal plane $(p<0.05$, Table 1). During STEP2 and STEP3, ankle energy generation was significantly 
higher compared to knee and hip joints in the sagittal plane $(p<0.05)$, but significantly smaller than the hip joint in the frontal plane $(p<0.05)$. During STEP2 and STEP3, the knee joint presented significantly smaller energy generation than the hip joint in both planes $(p<0.05)$.

Energy Differences between Planes

ANOVA results showed that the frontal plane had significantly higher energy absorption than the sagittal plane $(p<0.001)$, whereas the sagittal plane had significantly higher energy generation than the frontal plane $(p<0.001)$.

Sagittal Plane Energy

There were no sagittal plane energy data for the knee joint during STEP1. Ankle energy absorption (A1) was present in all three steps and modified as a function of the step number $(\mathrm{F}(2,42)$ $=36.27, p<0.0001)$ : A1 progressively increased in magnitude with each step. Ankle push-off (A2) was also present in all three steps, and modified as a function of the step number $(\mathrm{F}(2,42)=68.27, p$ $<0.0001)$ : A2 was lowest in STEP1, highest in STEP2, and all steps were different from each other.
At the knee, no knee energy bursts were apparent in STEP1, K2 and K4 appeared in STEP2, while STEP3 contained K2, K3 and K4. Therefore, at the knee, steady state gait patterns were achieved progressively with each step. Comparisons across the latter two steps demonstrated that only $\mathrm{K} 4$ was different $(\mathrm{F}(1,28)=$ 5.50, $p=0.026$ ), with STEP3 larger than STEP2.

At the hip, only hip pull-off (H3) was apparent at the toe-off in STEP1. Additional energy bursts became apparent as steps progressed: $\mathrm{H} 1$ was added at STEP2, and $\mathrm{H} 2$ was added at STEP3. H1 was significantly greater in STEP3 compared to STEP2 $(\mathrm{F}(1,26)=10.44, p=$ 0.0033). Hip pull-off (H3) was not different across the different steps $(\mathrm{F}(2,42)=2.32, p=0.11)$.

Frontal Plane Energy

There was no frontal plane energy data for the hip joint during STEP1. Only one frontal plane energy, $\mathrm{H} 2$, demonstrated a significant effect of the step number $(\mathrm{F}(1,28)=21.94, p<$ 0.0001 ) with post hoc analyses revealing that STEP2 was greater than STEP3.

Table 1

Relationships of support, hip, knee, and ankle moments in the sagittal and frontal planes

\begin{tabular}{|c|c|c|c|c|c|c|}
\hline \multicolumn{7}{|c|}{ Sagittal vs Frontal } \\
\hline Moment & & STEP1 & & STEP2 & & STEP3 \\
\hline Support & $-0.82^{* *}$ & (very high) & $0.80^{* *}$ & (very high) & $0.93^{* *}$ & (nearly perfect) \\
\hline Hip & $-0.45^{* *}$ & (moderate) & -0.16 & (low) & $-0.34^{* *}$ & (moderate) \\
\hline Knee & $0.32^{* *}$ & (moderate) & $-0.23^{*}$ & (low) & 0.10 & (low) \\
\hline Ankle & $-0.92^{* *}$ & (nearly perfect) & $0.87^{* *}$ & (very high) & $0.68^{* *}$ & (high) \\
\hline
\end{tabular}

** Correlation is significant at the $p<0.01$ level

* Correlation is significant at the $p<0.05$ level 
Table 2

Comparison of energy absorbed and generated during gait initiation

\begin{tabular}{lccc}
\hline & $\begin{array}{c}\text { STEP1 } \\
(\mathrm{J} / \mathrm{kg})\end{array}$ & $\begin{array}{c}\text { STEP2 } \\
(\mathrm{J} / \mathrm{kg})\end{array}$ & $\begin{array}{c}\text { STEP3 } \\
(\mathrm{J} / \mathrm{kg})\end{array}$ \\
\hline $\begin{array}{l}\text { Absorption* } \\
\text { Sagittal plane }\end{array}$ & & & \\
$-\quad$ Hip & -- & -- & $-2.18(2.16)$ \\
Knee & -- & $-3.92(1.29)^{3}$ & $-5.67(2.59)^{\mathrm{H}}$ \\
Ankle & $-0.88(0.56)^{2,3}$ & $-3.42(1.54)^{3}$ & $-4.54(1.30)^{\mathrm{H}}$ \\
Frontal plane & & & \\
Hip & -- & & $-0.48(0.30)$ \\
Knee & -- & $-0.62(0.30)$ & $-0.27(0.38)$ \\
Ankle & $-0.04(0.04)$ & $-0.73(0.58)^{3}$ & $-0.05(0.60)^{\mathrm{H}}$ \\
\hline
\end{tabular}

\section{Generation*:}

Sagittal plane

Hip

Knee

$4.52(1.48)^{3}$

$5.98(1.87)$

$6.72(2.77)$

Ankle

$0.40(0.51)^{2,3, \mathrm{H}}$

$2.88(1.45)^{3, \mathrm{H}}$

$1.45(1.20)^{\mathrm{H}}$

$17.69(6.27)^{3, K, H}$

$10.77(3.22)^{\mathrm{K}, \mathrm{H}}$

Frontal plane

$\begin{array}{lccc}\text { Hip } & -- & 5.25(1.14)^{3} & 2.80(1.68) \\ \text { Knee } & 0.18(0.23)^{2} & 0.56(0.27)^{\mathrm{H}} & 0.51(0.61)^{\mathrm{H}} \\ \text { Ankle } & 0.03(0.02)^{2} & 0.09(0.08)^{\mathrm{H}} & 0.05(0.05)^{\mathrm{H}}\end{array}$

$0.09(0.08)^{\mathrm{H}}$

$0.05(0.05)^{\mathrm{H}}$

Note: ${ }^{*}=$ significant difference between energy type, ${ }^{2}=$ significantly different from $S T E P 2,{ }^{3}=$ significantly different from STEP3, $\mathrm{K}=$ significantly different from the knee joint, ${ }^{H}=$ significantly different from the hip joint.

Table 3

Relationships of hip, knee, and ankle powers in the sagittal and frontal planes

\begin{tabular}{c|cc|cccc}
\hline \multicolumn{7}{c}{ Sagittal vs Frontal } \\
\hline & \multicolumn{2}{c}{ STEP1 } & \multicolumn{2}{c}{ STEP2 } & \multicolumn{2}{c}{ STEP3 } \\
\hline Hip & $-0.42^{* *}$ & (moderate) & $0.30^{* *}$ & $($ low $)$ & -0.02 & (trivial) \\
Knee & -0.01 & (trivial) & $-0.54^{* *}$ & (moderate) & $0.38^{* *}$ & (moderate) \\
Ankle & $0.72^{* *}$ & (high) & $0.89^{* *}$ & (very high) & $0.91^{* *}$ & (very high) \\
\hline
\end{tabular}

${ }^{* *}$ Correlation is significant at the $p<0.01$ level 


\section{Table 4}

Measurement reliability and variance

\begin{tabular}{|c|c|c|c|c|c|c|c|}
\hline \multicolumn{4}{|c|}{ Sagittal } & & \multicolumn{3}{|c|}{ Frontal } \\
\hline Hip: & ICC & $C V$ & $S D$ & & ICC & $C V$ & $S D$ \\
\hline L1H3 & 0.763 & $32.7 \%$ & $27.0 \%$ & L3H1 & 0.584 & $65.7 \%$ & $49.4 \%$ \\
\hline L3H1 & 0.736 & $53.7 \%$ & $35.1 \%$ & L3H2 & 0.966 & $30.1 \%$ & $25.1 \%$ \\
\hline L3H2 & 0.991 & $31.1 \%$ & $15.8 \%$ & $\mathrm{R} 2 \mathrm{H} 1$ & 0.707 & $57.5 \%$ & $37.8 \%$ \\
\hline L3H3 & 0.936 & $22.1 \%$ & $14.6 \%$ & $\mathrm{R} 2 \mathrm{H} 2$ & 0.815 & $17.7 \%$ & $9.0 \%$ \\
\hline $\mathrm{R} 2 \mathrm{H} 1$ & 0.808 & $37.6 \%$ & $30.2 \%$ & $\mathrm{R} 2 \mathrm{H} 3$ & 0.910 & $24.2 \%$ & $10.4 \%$ \\
\hline $\mathrm{R} 2 \mathrm{H} 3$ & 0.772 & $40.0 \%$ & $33.9 \%$ & & & & \\
\hline Average & 0.834 & $36.2 \%$ & & Average & 0.796 & $39.0 \%$ & \\
\hline Knee: & ICC & $C V$ & $S D$ & & ICC & $C V$ & $S D$ \\
\hline L3K2 & 0.774 & $42.3 \%$ & $24.2 \%$ & L1K1 & 0.951 & $65.7 \%$ & $27.6 \%$ \\
\hline L3K3 & 0.684 & $60.7 \%$ & $45.6 \%$ & L3K1 & 0.967 & $52.8 \%$ & $27.4 \%$ \\
\hline L3K4 & 0.959 & $22.8 \%$ & $9.5 \%$ & L3K2 & 0.970 & $51.9 \%$ & $28.8 \%$ \\
\hline $\mathrm{R} 2 \mathrm{~K} 2$ & 0.937 & $35.1 \%$ & $29.5 \%$ & $\mathrm{R} 2 \mathrm{~K} 1$ & 0.891 & $48.2 \%$ & $42.6 \%$ \\
\hline \multirow[t]{3}{*}{ R2K4 } & 0.837 & $26.7 \%$ & $13.0 \%$ & $\mathrm{R} 2 \mathrm{~K} 2$ & 0.919 & $45.4 \%$ & $22.9 \%$ \\
\hline & & & & $\mathrm{R} 2 \mathrm{~K} 3$ & 0.983 & $56.4 \%$ & $45.3 \%$ \\
\hline & & & & R2K4 & 0.936 & $32.8 \%$ & $16.8 \%$ \\
\hline Average & 0.838 & $37.5 \%$ & & Average & 0.945 & $50.5 \%$ & \\
\hline Ankle & ICC & $C V$ & $S D$ & & ICC & $C V$ & $S D$ \\
\hline L1A1 & 0.915 & $48.5 \%$ & $27.7 \%$ & L1A1 & 0.928 & $65.1 \%$ & $44.9 \%$ \\
\hline L1A2 & 0.938 & $89.6 \%$ & $62.2 \%$ & L1A2 & 0.925 & $64.7 \%$ & $42.0 \%$ \\
\hline L3A1 & 0.874 & $23.3 \%$ & $14.7 \%$ & L3A1 & 0.830 & $87.5 \%$ & $32.4 \%$ \\
\hline L3A2 & 0.948 & $14.4 \%$ & $6.6 \%$ & L3A2 & 0.852 & $111.3 \%$ & $52.5 \%$ \\
\hline R2A1 & 0.927 & $30.9 \%$ & $21.7 \%$ & R2A1a & 0.964 & $52.4 \%$ & $37.3 \%$ \\
\hline \multirow[t]{3}{*}{ R2A2 } & 0.950 & $19.8 \%$ & $12.1 \%$ & $\mathrm{R} 2 \mathrm{~A} 2 \mathrm{a}$ & 0.867 & $86.4 \%$ & $38.9 \%$ \\
\hline & & & & $\mathrm{R} 2 \mathrm{~A} 1 \mathrm{~b}$ & 0.745 & $93.7 \%$ & $29.5 \%$ \\
\hline & & & & $\mathrm{R} 2 \mathrm{~A} 2 \mathrm{~b}$ & 0.774 & $95.0 \%$ & $49.9 \%$ \\
\hline Average & 0.925 & $37.8 \%$ & & Average & 0.861 & $82.0 \%$ & \\
\hline
\end{tabular}

$I C C=$ Intraclass correlation coefficient $; C V=$ Coefficient of variation; $S D=$ Standard deviation Ln = Left STEPn; Rn=Right STEPn; $A=$ Ankle; K = Knee; $H=$ Hip 


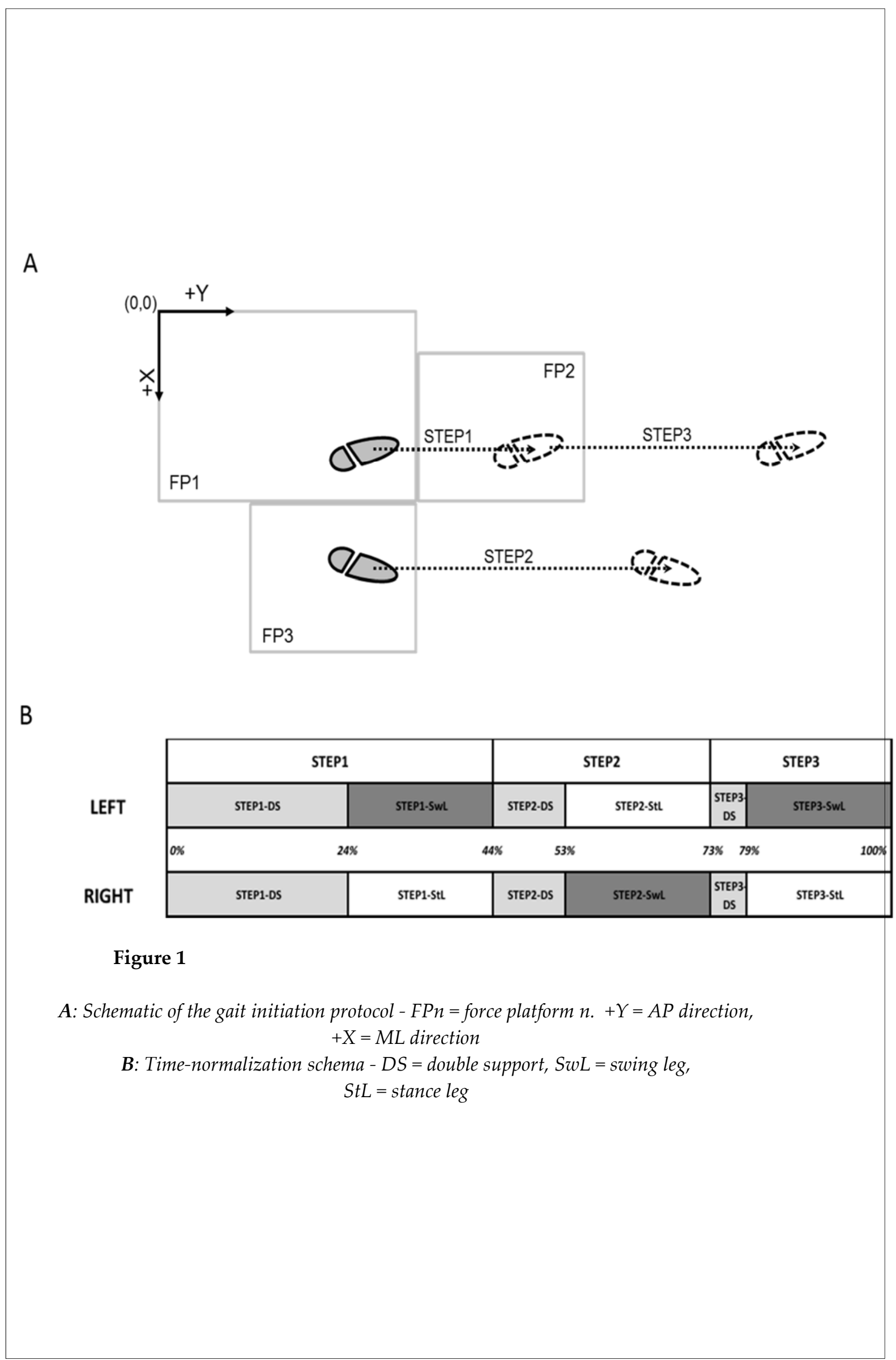

(c) Editorial Committee of Journal of Human Kinetics 


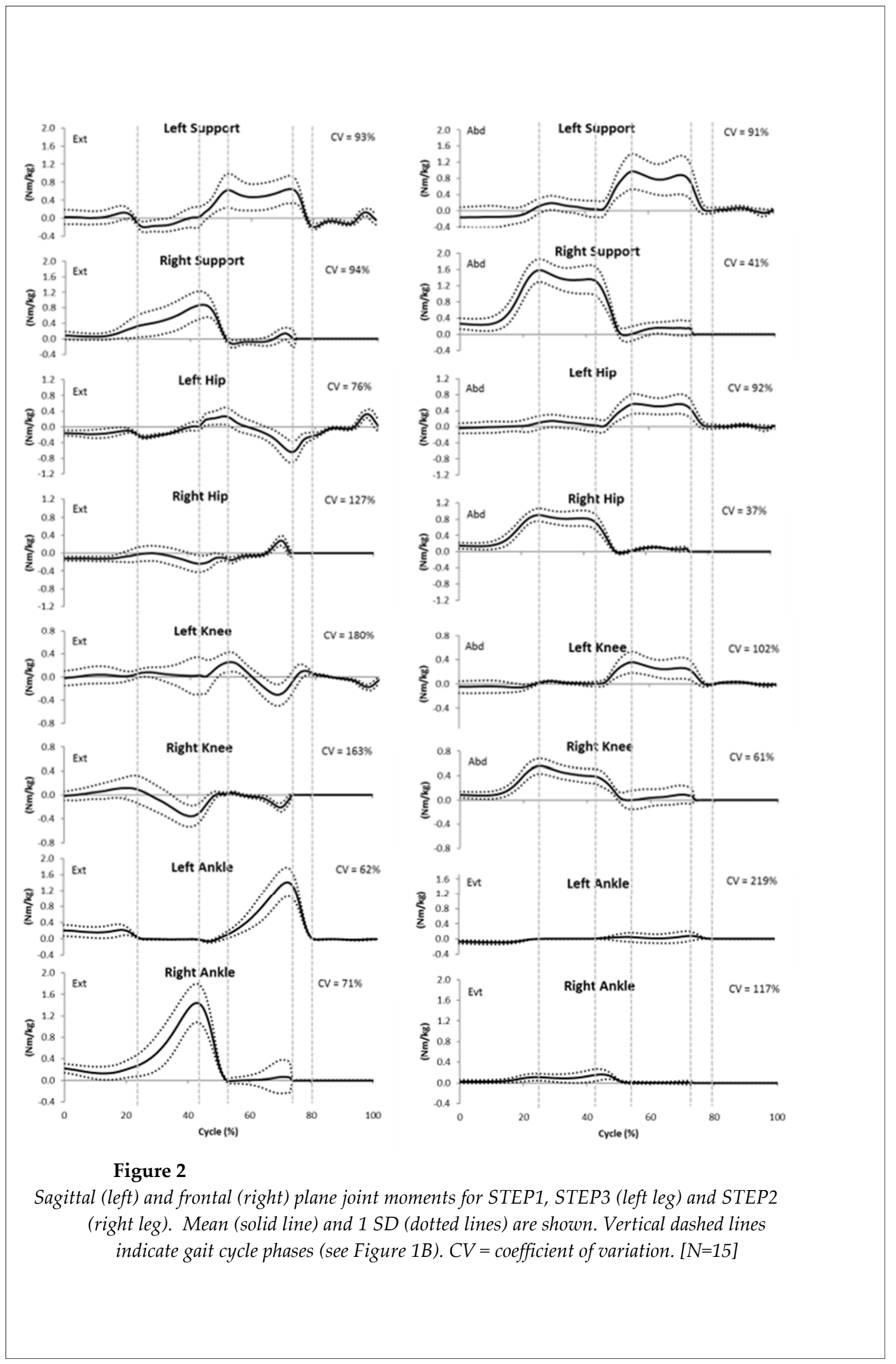




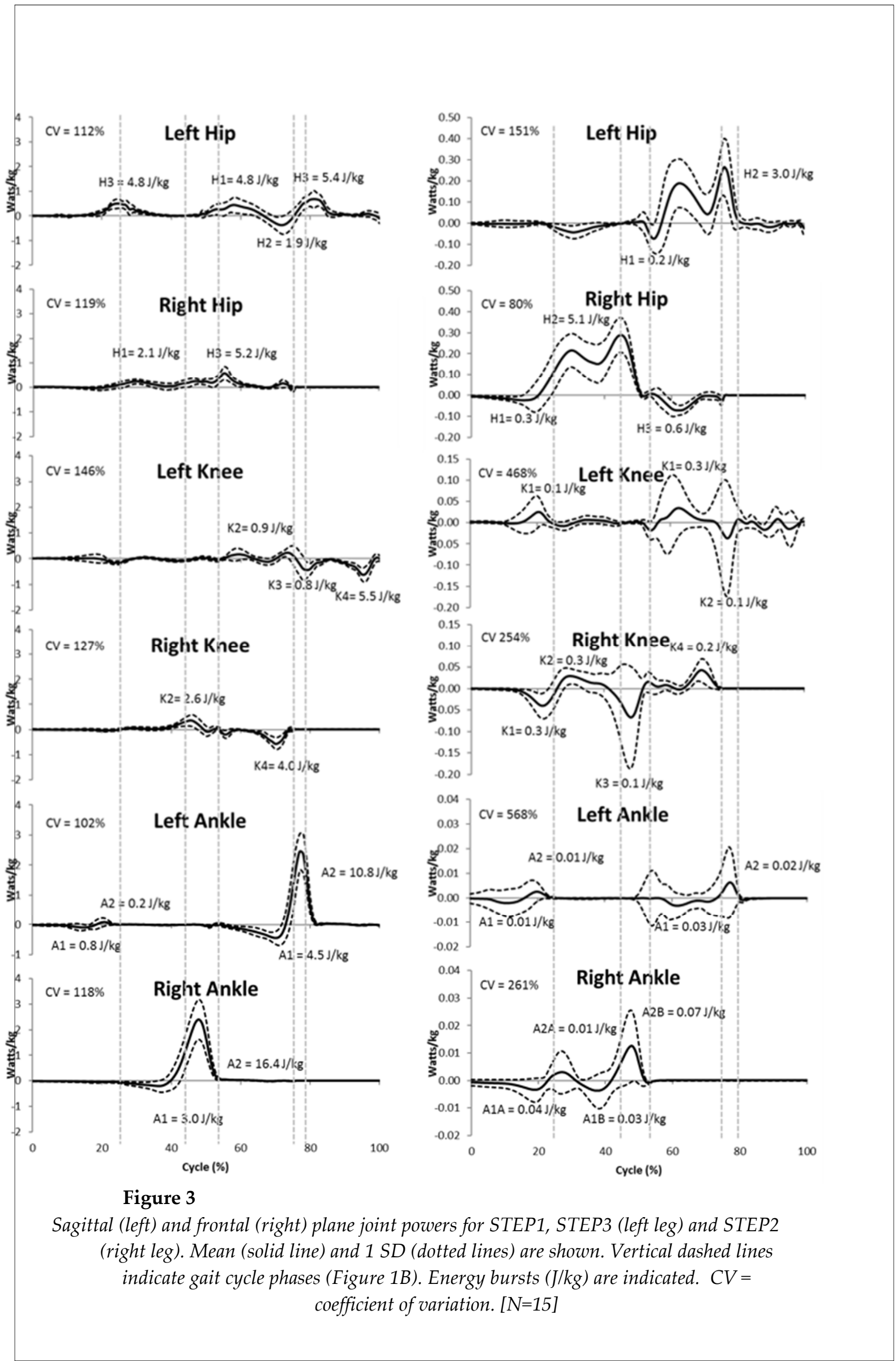

(c) Editorial Committee of Journal of Human Kinetics 


\section{Joint Power Planar Comparisons}

Joint power planar correlations are shown in Table 2 and the ankle shows consistently high to nearly perfect positive correlations between planes, while the hip in STEP1 and the knee in STEP2 and STEP3 have moderate correlations.

\section{Test-retest Reliability and Variance}

The ICC results along with CV data are presented in Table 3. Overall, the average ICC values are very high, ranging from 0.834 to 0.925 for the sagittal plane energy data and from 0.796 to 0.945 for the frontal plane energy data, indicating that the presence of these energy bursts is quite repeatable. The CV results for the sagittal plane energy data are consistent, averaging between 36.6 and $37.5 \%$. On the other hand, the $\mathrm{CV}$ data for the frontal plane are more variable, averaging from 39.0 to $82.0 \%$, with the ankle showing particularly high variability (range: 52.4 to $111.3 \%$ ). In general, for the frontal plane, the more distal the joint, the higher the variation between subjects.

\section{COM Kinematics}

The velocity and acceleration of the COM were calculated for each step. COM velocity revealed an increasing pattern from STEP1 $(0.83 \pm$ $\left.0.15 \mathrm{~m} \cdot \mathrm{s}^{-1}\right)$ through STEP2 $\left(1.10 \pm 0.18 \mathrm{~m} \cdot \mathrm{s}^{-1}\right)$ to STEP3 $\left(1.16 \pm 0.14 \mathrm{~m} \cdot \mathrm{s}^{-1}\right)$ with the velocity of STEP1 being significantly lower than both STEP2 and STEP3 $(\mathrm{F}=16.3, p<0.001)$. For COM acceleration, the pattern was different, with STEP2 $\left(1.34 \pm 0.30 \mathrm{~m} \cdot \mathrm{s}^{-2}\right)$ being smaller than both STEP1 $\left(1.62 \pm 0.37 \mathrm{~m} \cdot \mathrm{s}^{-2}\right)$ and STEP3 $(1.45 \pm 0.65$ $\left.\mathrm{m} \cdot \mathrm{s}^{-2}\right)$. COM acceleration of STEP2 was significantly smaller compared to STEP1 (F-4.44, $p$ $<0.04)$. The reliability of the COM kinematics was nearly perfect, with ICC values ranging from 0.95 to 0.98 and $C V$ values $\leq 20.0 \%$.

\section{Discussion}

Transition from standing to walking is not an insignificant event. Goals during QS include maintaining support by locking the hip and knee joints, while allowing small motions at the ankle as the COP contains the COM within the base of support. In the case of a steady-state gait, the COP can move beyond the base of support as the function of each limb shifts from supporting the body while first absorbing, then generating energy, to swinging forward ahead of the body in preparation for the next step. During the single support period of stance, one of the goals is the same as QS: keep the COM within the base of support, i.e., the stance foot, but the motion of the COM is predominantly in the forward direction. As a result, accelerations are much larger than during QS, with commensurate increases in joint moments and energy.

Joint, Support, and Centering Moments

When first proposed by Winter (1980), the principle of the support moment was an attempt to explain how, in some cases, repeatable lower extremity joint moment patterns at the hip and knee were $180^{\circ}$ out of phase as compared to most healthy, normal subjects; yet these subjects otherwise demonstrated successful gait patterns. In particular, some subjects presented with a flexor knee moment during single support in a stance, the implication of which is that, from the perspective of the knee alone, this should have meant the leg was collapsing. However, by considering the entire limb as a single unit, the principle of the support moment allowed for the individual joints of the lower extremity to differ considerably from subject to subject, so long as there was a sufficiently large extensor support moment during stance.

At the time that this principle was proposed, most gait analyses only considered motion in the sagittal plane, so it was natural for Winter to see the goal of the support moment in terms of the behavior of the lower extremity joints in that plane, i.e., resisting collapse of the body, particularly in single support. Since the support moment was first proposed, 3D gait analysis has become the standard in virtually all motion analysis laboratories. This has allowed for examination of sagittal, frontal, and transverse gait kinematics and kinetics. However, to date, there has been no examination of the 3D manifestation of the support moment and what role(s) it may play in the frontal plane. This study was an attempt to explore this phenomenon more fully.

The results of this study (Figure 2) clearly indicate that the typical support moment pattern does not emerge until the STEP2 in both the sagittal and frontal planes. Since the motion in the early part of STEP1 is predominately lateral in direction, it is not surprising that the right leg frontal plane support moment (STEP2) begins even before the left foot has left the ground. The 
magnitude of the frontal plane support moment in STEP2 is much larger compared to STEP3, which is also consistent with the lateral-thenanterior motion of the COM during the first two steps of GI. By STEP3, it is reasonable to assume that the support moment patterns are fully developed in both planes, which is supported by our findings.

The patterns of joint moments in the sagittal plane also display the gradual development over each step towards that seen in steady-state gait. On one hand, what is most interesting is that while the dominant joint moment occurs at the ankle in the sagittal plane, it is the hip that has the largest moments in the frontal plane, with decreasing magnitudes in the knee and ankle, respectively. On the other hand, there are virtually no adductor or invertor moments occurring in the frontal plane. As such, joint patterns at the hip and knee resemble the abductor support moment. This is the first clue that the goals of the support moment in the sagittal and frontal planes may not be identical. In STEP2 and STEP3, the ankle has the largest contribution to the extensor nature of the sagittal support moment as motion in the sagittal plane dominates with the plantarflexor muscles providing most of the energy needed. Simultaneously during the same steps, in the frontal plane, the COM moves laterally to its limit, then moves back towards the midline, with the hip abductors providing the trunk stabilization needed to facilitate this motion.

The correlation data in Table 1 give some insight into how the sagittal and frontal support moments are related. Positive correlations indicate that the support moment is acting to both prevent collapse in the sagittal plane (extensor) and to keep the COM close to the path of motion near the midline of the body (abductor), preventing excessive lateral motion. At the joints, positive correlations indicate both extensor and abductor, or plantarflexor and evertor, moments occur together. In STEP1, three of the four correlations are negative, which is expected since the participants do not want to collapse, but want to allow for movement of the COM in the frontal plane to unweight the STEP1 supporting limb, then return the COM to the midline position during the step. In STEP2 and STEP3, the correlations are similar whereby the support moment and ankles have high and very high positive correlations, suggesting that resisting collapse and keeping the COM close to the midline are the goals, as the pattern of steadystate gait becomes increasingly evident. Just as Winter (1980) saw trade-offs between the hip and knee moments in the sagittal plane, there is evidence in our data of planar trade-offs at the hip and knee joints (Table 1).

If the overall sagittal plane extensor pattern of the support moment gave rise to its support role and moniker, what is to be made of the different role of the summation of abductor/evertor moments in the frontal plane? These exist to resist another threat to successful gait, but it is not to prevent collapse, but rather lateral falls. Thus, taking the frontal plane into consideration, we propose to extend Winter's support moment principle to include both a support, or anti-gravity, component in the sagittal plan as well as a centering component in the frontal plane.

Joint Energies

From the data shown in Figure 3, it would seem that very little energy is absorbed at the ankle during the dorsiflexion that occurs in stance in either STEP1 or STEP2. Instead, there seems to be considerable energy generated at the hip (STEP1 and STEP2) and the knee (STEP2).

We also know that during steady-state gait, the plantarflexors act eccentrically during early stance to control the forward rotation of the shank, as evidenced by the negative energy phase typically labelled A1 (Winter, 1983b). Miller and Verstraete (1999) found that the greatest increase in energy occurred in STEP2, and in our data STEP2 at virtually all joints and both planes had the largest energy values. However, the same step had the lowest resultant acceleration of the COM. If the goal of GI is to achieve steady-state velocity as soon as safely possible, then one can imagine an initial burst of acceleration (from both muscular and inertial sources) during STEP1 to get things started (similar to putting one's foot on the car's accelerator), then reduced acceleration in STEP2 so that you do not 'over-shoot' the desired velocity (lifting your foot off the accelerator slightly), then, if necessary, adding slightly more acceleration in STEP3 as we 'fine-tune' the steadystate gait velocity profile.

To date, there exist far fewer published 
studies related to frontal plane kinetics in steadystate gait and none specifically investigating GI. Eng and Winter (1995) reported sagittal and frontal plane lower limb moments and powers during steady-state gait from nine male subjects. Similarity in shape, and to a certain extent, magnitudes between their data and ours show general agreement for the hip and knee for all steps. However, while the sagittal ankle moments have some similarities, the frontal plane powers at the ankle are quite different in that where our data show energy generation during push-off, the data of Eng and Winter (1995) indicate energy absorption during steady-state gait. The authors do note that their frontal plane ankle power data were small and highly variable, which our study corroborates.

What may be more interesting in comparing joint power patterns between planes (Table 3) is the fact that the ankle joint appears to be acting synchronously with positive correlations increasing from 0.72 (STEP1) to 0.91 (STEP3). In addition, the hip in STEP1 and the knee in STEP2 have moderate negative correlations of -0.42 and 0.54 respectively, suggesting the possibility that energy flows may occur between planes.
The present study has documented the sagittal and frontal plane joint and support/centering moments, and joint powers and energies during the first three steps of GI in a sample of healthy, young adults. The methods of movement analysis used in this study offer powerful mechanisms to understand the crucial transition from QS to purposeful, and often highly dynamic, human movement including steadystate gait, as well as sporting activities such as running and jumping events (long jump, high jump, triple jump).

For the most part, our hypotheses were supported; however, differences in the power profile at the ankle joint as compared to published data on steady-state gait suggest that further study comparing these two activities is warranted. In addition, further exploration of how this centering moment manifests itself in a wide range of locomotion activities, including highly skilled performers such as dancers, gymnasts, and tai chi practitioners, may make a significant contribution to research into freeze of gait episodes in people with Parkinson's disease (Delval et al., 2014), and fall prevention, particularly when the person is moving laterally and when negotiating turns (Cumming and Klineberg, 1994).

\section{Acknowledgements}

The authors wish to acknowledge the contribution by Dr. Shirley Rietdyk of the Purdue University to discussions of the interpretation of the data.

\section{References}

Breniere Y, Do MC. When and how does steady state gait movement induced from upright posture begin? J Biomech, 1986; 19: 1035-1040

Brown NA, Pandy MG, Kawcak CE, McIlwraith CW. Force- and moment-generating capacities of muscles in the distal forelimb of the horse [Online]. J Anat 2003; 203: 101-113 http://www.ncbi.nlm.nih.gov/pmc/articles/PMC1571149/pdf/joa0203-0101.pdf

Brunt D, Liu SM, Trimble M, Bauer J, Short M. Principles underlying the organization of movement initiation from quiet stance. Gait Posture 1999; 10: 121-128

Brunt D, Short M, Trimble M, Liu SM. Control strategies for initiation of human gait are influenced by accuracy constraints. Neurosci Lett 2000; 285: 228-230

Burton NJ, Owen MR, Kirk LS, Toscano MJ, Colborne GR. Conservative versus arthroscopic management for medial coronoid process disease in dogs: a prospective gait evaluation. Vet Surg 2011; 40: 972-980

Callisaya ML, Blizzard L, Martin K, Srikanth VK. Gait initiation time is associated with the risk of multiple falls-A population-based study. Gait Posture 2016; 49: 19-24

Carlsöö S. The initiation of walking. Acta Anat (Basel) 1966; 65: 1-9

Chmielewski TL, Rudolph KS, Fitzgerald GK, Axe MJ, Snyder-Mackler L. Biomechanical evidence supporting a differential response to acute ACL injury [Online]. Clin Biomech (Bristol, Avon) 2001; 16: 
586-591 http://www.clinbiomech.com/article/S0268-0033(01)00050-X/abstract

Corbeil P, Anaka E. Combined effects of speed and directional change on postural adjustments during gait initiation. J Electromyogr Kinesiol Off J Int Soc Electrophysiol Kinesiol 2011; 21: 734-741

Crenna P, Frigo C. A motor programme for the initiation of forward-oriented movements in humans. $J$ Physiol 1991; 437: 635-653

Cumming RG, Klineberg RJ. Fall frequency and characteristics and the risk of hip fractures. J Am Geriatr Soc 1994; 42: 774-778

Delval A, Tard C, Defebvre L. Why we should study gait initiation in Parkinson's disease. Neurophysiol Clin Neurophysiol 2014; 44: 69-76

Eng JJ, Winter DA. Kinetic analysis of the lower limbs during walking:what information can be gained from a three-dimensional model? J Biomech 1995; 28: 753-757

Halliday SE, Winter DA, Frank JS, Patla AE, Prince F. The initiation of gait in young, elderly, and Parkinson's disease subjects. GaitPosture 1998; 8: 8-14

Hebert LJ, Gravel D, Arsenault AB, Tremblay G. Patellofemoral pain syndrome: the possible role of an inadequate neuromuscular mechanism. Clin Biomech 1994; 9: 93-97

Hong SW, Leu TH, Li JD, Wang TM, Ho WP, Lu TW. Influence of inclination angles on intra- and inter-limb load-sharing during uphill walking. Gait Posture 2014a; 39: 29-34

Hong SW, Wang TM, Lu TW, Li JD, Leu TH, Ho WP. Redistribution of intra- and inter-limb support moments during downhill walking on different slopes. J Biomech 2014b; 47: 709-715

Hopkins WG. Measures of reliability in sports medicine and science [Online]. Sport Med 2000; 30: 1-15 http://www.ncbi.nlm.nih.gov/pubmed/10907753.

Hwang S, Kim Y. Lower extremity joint kinetics and lumbar curvature during squat and stoop lifting. BMC Musculoskelet Disord 2009; 10: 15

Jian Y, Winter D, Ishac M, Gilchrist LA. Trajectory of the body COG and COP during initiation and termination of gait. Gait Posture 1993; 1: 9-22

Lay AN, Hass CJ, Gregor RJ. The effects of sloped surfaces on locomotion: a kinematic and kinetic analysis. J Biomech 2006; 39: 1621-1628

Liu MF, Chou PH, Liaw LJ, Su FC. Lower-limb adaptation during squatting after isolated posterior cruciate ligament injuries. Clin Biomech 2010; 25: 909-913

Mandeville D, Osternig LR, Chou LS. The effect of total knee replacement on dynamic support of the body during walking and stair ascent. Clin Biomech 2007; 22: 787-794

Mann RA, Hagy JL, White V, Liddell D. The initiation of gait. JBone Jt SurgAm 1979; 61: 232-239

McCaw ST, Gardner JK, Stafford LN, Torry MR. Filtering ground reaction force data affects the calculation and interpretation of joint kinetics and energetics during drop landings. J Appl Biomech 2013; 29: 804809

McFadyen BJ, Carnahan H. Anticipatory locomotor adjustments for accommodating versus avoiding level changes in humans. Exp Brain Res 1997; 114: 500-506

McNee AE, Shortland AP, Eve LC, Robinson RO, Gough M. Lower limb extensor moments in children with spastic diplegic cerebral palsy. Gait Posture 2004; 20: 171-176

McQuade KJ, de Oliveira AS. Effects of progressive resistance strength training on knee biomechanics during single leg step-up in persons with mild knee osteoarthritis. Clin Biomech 2011; 26: 741-748

Miff SC, Hansen AH, Childress DS, Gard SA, Meier MR. Roll-over shapes of the able-bodied knee-ankle-foot system during gait initiation, steady-state walking, and gait termination. Gait Posture 2008; 27: 316-322

Miller CA, Verstraete MC. Determination of the step duration of gait initiation using a mechanical energy analysis. J Biomech 1999a; 29: 1195-1199

Miller CA, Verstraete MC. A mechanical energy analysis of gait initiation. Gait Posture 1999b; 9: 158-166

Muir BC, Rietdyk S, Haddad JM. Gait initiation: The first four steps in adults aged 20-25 years, 65-79 years,

(c) Editorial Committee of Journal of Human Kinetics 
and 80-91 years. Gait Posture 2014; 39: 490-494

Novak AC, Brouwer B. Sagittal and frontal lower limb joint moments during stair ascent and descent in young and older adults. Gait Posture 2011; 33: 54-60

Novak AC, Brouwer B. Kinematic and kinetic evaluation of the stance phase of stair ambulation in persons with stroke and healthy adults: a pilot study. J Appl Biomech 2013; 29: 443-452

Orishimo KF, Kremenic IJ. Effect of fatigue on single-leg hop landing biomechanics. J Appl Biomech 2006; 22: $245-254$

Perell KL, Gregor S, Kim G, Rushatakankovit S, Scremin E, Levin S, Gregor R. Comparison of cycling kinetics during recumbent bicycling in subjects with and without diabetes. J Rehabil Res Dev 2002; 39: 13-20

Rogers MW, Hedman LD, Johnson ME, Cain TD, Hanke TA. Lateral stability during forward-induced stepping for dynamic balance recovery in young and older adults. Journals Gerontol Ser A Biol Sci Med Sci 56: 2001; M589-M594

Rogers MW, Mille M-LL. Lateral stability and falls in older people. Exerc Sport Sci Rev 2003; 31: 182-187

Roos PE, Button K, van Deursen RW. Motor control strategies during double leg squat following anterior cruciate ligament rupture and reconstruction: an observational study. J Neuroeng Rehabil 2014; 11: 19

Sanderson DJ, Martin PE. Joint kinetics in unilateral below-knee amputee patients during running [Online]. Arch Phys Med Rehabil 1996; 77: 1279-1285 http://www.archives-pmr.org/article/S0003-9993(96)901938/pdf.

Seeley MK, Park J, King D, Hopkins JT. A novel experimental knee-pain model affects perceived pain and movement biomechanics. J Athl Train 2013; 48: 337-345

Veldpaus FE, Woltring HJ, Dortmans LJ. A least-squares algorithm for the equiform transformation from spatial marker co-ordinates. J Biomech 1988; 21: 45-54

Wajda DA, Moon Y, Motl RW, Sosnoff JJ. Preliminary investigation of gait initiation and falls in multiple sclerosis. Arch Phys Med Rehabil 2015; 96: 1098-102

Winter DA. Overall principle of lower limb support during stance phase of gait [Online]. J Biomech 1980; 13: 923-927 http://www.jbiomech.com/article/0021-9290(80)90162-1/abstract.

Winter DA. Biomechanical motor patterns in normal walking. J Mot Behav 1983a; 15: 302-330

Winter DA. Energy generation and absorption at the ankle and knee during fast, natural, and slow cadences. ClinOrthop 1983b; 175: 147-154

Winter DA. Biomechanics and motor control of human movement. 2nd ed. New York: Wiley, 1990

\title{
Corresponding author:
}

\author{
Andrew W. Smith, PhD \\ Motion Analysis Research Center \\ Samuel Merritt University \\ 400 Hawthorne Avenue, Suite 101 \\ Oakland, CA 94609, USA \\ Tel.: +1-510-879-9242 \\ Fax: +1-510-457-2620 \\ E-mail: asmith3@samuelmerritt.edu
}

